\title{
Comparison Between Korean Version of Physical Activity Scale for the Elderly and International Physical Activity Questionnaire-Short Form in Evaluation of Frailty Phenotype
}

\author{
Il-Young Jang ${ }^{1,2, *}$, Hee-Won Jung ${ }^{3, *}$, Chang Ki Lee ${ }^{2,4}$, Young Soo Lee ${ }^{1}$, Eunju Lee ${ }^{1}$, Dae Hyun Kim ${ }^{5,6}$ \\ ${ }^{1}$ Department of Internal Medicine, Asan Medical Center, University of Ulsan College of Medicine, Seoul, ${ }^{2}$ Pyeongchang Health Center \\ \& County Hospital, Pyeongchang, ${ }^{3}$ Graduate School of Medical Science and Engineering, Korea Advanced Institute of Science and \\ Technology (KAIST), Daejeon, Korea, ${ }^{4}$ Goldman Urology Clinic, Seoul, ${ }^{5}$ Division of Gerontology, Department of Medicine, Beth Israel \\ Deaconess Medical Center, Boston, MA, ${ }^{6}$ Division of Pharmacoepidemiology and Pharmacoeconomics, Department of Medicine, Brigham \\ and Women's Hospital, Boston, MA, USA
}

Corresponding Author: Eunju Lee, $\mathrm{MD}, \mathrm{PhD}$ Department of Internal Medicine, Asan Medical Center, 88 Olympic-ro 43-gil, Songpa-gu, Seoul 05505, Korea

Tel: $+82-2-3010-3308$ Fax: +82-2-476-0824

E-mail: eunjulee@amc.seoul.kr *These authors contributed equally to this study and should be considered co-first authors.

Received: June 16, 2017 Revised: July 4, 2017 Accepted: July 5, 2017
Background: Evaluation of frailty in older people requires assessment of their physical activity level. The Korean version of the Physical Activity Scale for the Elderly (K-PASE) questionnaire is more comprehensive, yet it can be time-consuming and costly to administer on a large scale. The International Physical Activity Questionnaire (IPAO) short form, which is simpler and free of charge, may replace the K-PASE for defining the Cardiovascular Health Study (CHS) frailty phenotype. Methods: We analyzed data from 160 community-dwelling older adults (mean age: 75 years) who were administered both the K-PASE and IPAQ short form questionnaires as part of the assessments in the Aging Study of Pyeongchang Rural Area. We compared agreements between the 2 physical activity questionnaires and between the CHS frailty phenotype defined using the K-PASE and IPAO short form. Correlations between each CHS frailty phenotype definition and common geriatric syndromes were assessed. Results: The physical activity level measured using the K-PASE correlated modestly with the level measured using the IPAO short form (correlation coefficient, 0.250; $p=0.001$ ). However, the agreement was substantially higher between the CHS frailty phenotype based on the K-PASE and the definition based on the IPAO short form (Cohen kappa, $0.625 ; p<0.001)$. Both frailty phenotype definitions were positively correlated with most geriatric conditions. Conclusion: The simpler IPAQ short form may replace the more comprehensive K-PASE for assessing CHS frailty phenotype in older people. Our results should inform frailty assessment in research and clinical care settings.

Key Words: Frail elderly, Cohort studies, Exercise, Motor activity, Physical activity

\section{INTRODUCTION}

With the unprecedented trend of aging around the world, the importance of screening frailty in older people is ever increasing ${ }^{1)}$. Frailty is a consequence of aging defined by a decreased physiological reserve to various stressors ${ }^{2)}$. In other words, frailty can reflect the physiologic age of a person ${ }^{3)}$. Since frailty status can predict adverse health events in older people more effectively than do age and comorbidities $^{4)}$, accounting for frailty in therapeutic decision making is essential ${ }^{5}$. Therefore, evaluating frailty in older people has become a critical component of geriatric medicine and aging research.

To date, numerous measures have been proposed to assess frailty status in older people. In general, most tools can be categorized as either a phenotype model or an accumulationof-deficit model ${ }^{2,3,6)}$. Many clinical and preclinical studies used the Cardiovascular Health Study (CHS) frailty phenotype as a reference standard method ${ }^{7-10)}$. As the construct validity and predictive validity of the CHS frailty phenotype have also been demonstrated in older Koreans ${ }^{5}$, the CHS frailty phenotype is widely used for varying purposes in Korea ${ }^{8,11)}$.

Regarding the frailty phenotype, measuring physical activity level is necessary to define a low activity status. One of the most commonly used methods to measure this component is the Physical Activity Scale for the Elderly (PASE). Although the feasibility and construct validity of the Korean translated version of PASE (K-PASE) were established, its association with outcome measures has not been evaluated ${ }^{12)}$. 
There are barriers to administering the K-PASE in a large-scale population-based study owing to its proprietary right (which requires a license fee) and its length (which takes up to 10 minutes to administer). Furthermore, results from the PASE cannot be converted to a standardized quantity, such as the total metabolic equivalent task minutes per week (MET$\min / \mathrm{wk}$ ).

Alternatively, the Korean translated International Physical Activity Questionnaire (IPAQ) short form is a free tool that measures activity level in MET-min/wk. In older Koreans, the quantitative results calculated from the IPAQ short form was validated against the activity level obtained using an accelerometer ${ }^{13)}$. Owing to these advantages, the IPAO short form is currently used in nationwide studies, such as the Korea National Health And Nutrition Examination Survey $(\mathrm{KNHANES})^{14)}$ and the Korean Frailty and Aging Cohort Study $(\mathrm{KFACS})^{8)}$. Although the IPAQ short form may substitute K-PASE for identifying the CHS frailty phenotype in older Koreans, no studies have examined the agreement of the CHS frailty phenotype definitions based on the K-PASE and IPAQ short form.

In this study, we aimed to assess the correlations between the K-PASE and IPAQ short form in community-dwelling older people and the validity of substituting the K-PASE with the IPAQ short form for defining the CHS frailty phenotype.

\section{MATERIALS AND METHODS}

\section{Study Population}

The Aging Study of Pyeongchang Rural Area (ASPRA) is a population-based, prospective cohort study of frailty and geriatric syndromes in 1,350 older adults who are registered in the Community Healthcare Service and reside in Pyeongchang-gun, Gangwon-do, Korea. Detailed information on study design, target population, and measurements in ASPRA were published previously $y^{7)}$. People were eligible for the study if they were (1) age 65 years or older, (2) registered in the Community Healthcare Service, (3) ambulatory with or without an assistive device, (4) living at home, and (5) able to provide informed consent. We excluded those who were (1) living in a nursing home, (2) hospitalized, or (3) bedridden and receiving nursing home level care at home at the time of enrollment.

Participants were recruited between October 2014 and May 2017 and underwent annual comprehensive geriatric assessments that encompassed the assessment of cognitive and physical function, depression, nutrition, and body composition using bioimpedance analysis. The participation rate was $95 \%$, and the characteristics of ASPRA participants were comparable with those of a nationally representative sample of the Korean rural population ${ }^{7,15)}$, except that a higher pro- portion of ASPRA participants were working in agriculture and had a little formal education.

This analysis included 160 consecutive participants of ASPRA who were examined from November 2015 to December 2015 and were administered both the IPAO and K-PASE for the quantification of physical activity level. The protocol of this study was approved by the Institutional Review Board of the Asan Medical Center (IRB No. 2015-0673).

\section{Quantification of Physical Activity}

\section{1) The K-PASE}

The PASE is an instrument designed to assess physical activity in the previous week ${ }^{12,16,17)}$. This tool assesses type of leisure activities (walking, light, moderate, and strenuous sport activities, recreation and muscle strengthening exercise), paid work involving physical activities and participation on housework (lawn work, home repair, gardening, caring for others) in frequency (1-2 days/wk for seldom, 3-4 days/wk for sometimes, 5-7 days/wk for often) and duration ( $<1 \mathrm{hr}$, 1-2 hr, 2-4 hr, 4 or more hours). Predefined weights were applied to each activity to calculate a total physical activity level.

\section{2) The Korean translated IPAQ}

We used the short form of the IPAO to quantify physical activity levels. The IPAO short form ${ }^{13)}$ assesses the history of physical activity in the previous 7 days. We adopted the IPAO questionnaire used in KNHANES, which assessed the duration of 5 types of activities: (1) vigorous physical activity, (2) moderate physical activity, (3) walking, (4) number of days of resistance exercise, and (5) number of days of flexibility exercise. Among these variables, we used total duration (minutes) of vigorous activity, moderate physical activity, and walking to calculate total MET-min/wk. According to the IPAO scoring protocol, total physical activity level (MET-min/ wk) was calculated using the following equation: 8.0 METs $x$ total duration of vigorous activity + 4.0 METs $\times$ total duration of moderate activity +3.3 METs $\times$ total duration of walking.

\section{Frailty Assessment}

We used the CHS frailty phenotype scale ${ }^{6)}$. In this scale, frailty was defined as the presence of at least 3 of the following components: (1) exhaustion: an answer of "moderate or most of the time during the last week" to either of the following: "I felt that everything I did was an effort" or "I could not get going"; (2) low activity: physical activity level in the lowest quintile according to the K-PASE (below the 20th percentile cutoff point in the ASPRA cohort), IPAO (below the 20th percentile cutoff point in a representative sample of older 
Koreans in the KNHANES), or using a cutoff point derived from the regression analysis (described in the statistical analysis section below); (3) slowness: usual gait speed $<0.8 \mathrm{~m} / \mathrm{sec}$; (4) weakness: dominant handgrip strength $<26 \mathrm{~kg}$ for men and $<17 \mathrm{~kg}$ for women; and (5) weight loss: unintentional weight loss of $>3 \mathrm{~kg}$ during the previous 6 months ${ }^{5,18,19)}$.

\section{Assessment of Other Geriatric Conditions}

Trained nurses determined the existence of common geriatric conditions using the following instruments: the Korean version of the Mini-Mental State Examination (K-MMSE) for cognitive function, handgrip strength and usual gait speed for physical performance, the Korean version of the Center for Epidemiological Studies Depression (CES-D) scale for depressive mood, the Mini-Nutritional Assessment-Short Form (MNA-SF) score for malnutrition, and the Korean activities of daily living (ADL) and Korean instrumental ADL for dependence, with the previously validated cutoff points ${ }^{7)}$. Sarcopenia was defined according to the Asian Working Group on Sarcopenia (AWGS) ${ }^{20)}$ criteria, based on bioimpedance analysis (Inbody 620; Inbody, Seoul, Korea), usual gait speed, and handgrip strength (T.K.K. 5401 Grip-D; Takei, Tokyo, Japan). Low cognition was defined as a K-MMSE score of $<24^{21)}$, and high depressive symptoms were defined as a CES-D score of $\geq 21^{222}$. We defined a risk for malnutrition as an MNA-SF score of $\leq 11$ points $^{23}$. These conditions were used to test the face validity of physical activity questionnaires and frailty definitions.

\section{Statistical Analysis}

We summarized the characteristics of the study population using the mean, standard deviation (SD), and proportion. The distribution of and Spearman correlation between total physical activity levels quantified using the K-PASE and IPAO short form were examined. To compare the face validity of physical activity questionnaires, we calculated Spearman correlation coefficients of each physical activity scale with common geriatric conditions. To evaluate the impact of choice of physical activity scales on the frailty definition, we created separate CHS frailty definitions based on how low activity was defined: frailty using the population-specific cutoff point of the K-PASE in the ASPRA cohort (CHS-PASE) and frailty using the population-independent cutoff point of the IPAQ short form (CHS-IPAQ). The population-independent 20th percentile cutoff point of the IPAQ short form was estimated using data from 8,018 older people in the 6th KNHANES dataset. We then assessed the Cohen kappa agreement and Spearman correlation coefficient between the CHS-PASE and CHS-IPAO. In addition, since the IPAO short form is used to measure physical activity level in KNHANES, we derived a conversion equation that used K-PASE items to predict a low physical activity state defined using the IPAO short form; this conversion enables a direct comparison of frailty status defined using the K-PASE and IPAO short form. To develop the conversion equation, we first fitted logistic regression to model a low physical activity state defined by the IPAO short form as a function of individual K-PASE items. The probability of having a low physical activity level was estimated from the logistic model and a cutoff point was chosen such that it maximized the Youden index ${ }^{24)}$. We defined CHS frailty status (CHS-IPAQ PASE) using the predicted low physical activity state and assessed the Cohen kappa agreement and Spearman correlation coefficient between CHS-IPAQPASE and CHS-IPAQ. To assure whether replacing the K-PASE with the IPAQ short form in defining frailty can be justified, we evaluated the Spearman correlation between each frailty definition and geriatric conditions. Statistical analysis was carried out using IBM SPSS Statistics ver. 21.0 (IBM Co., Armonk, NY, USA). A 2-sided p-value $<0.05$ was considered statistically significant.

\section{RESULTS}

\section{Characteristics of Study Population}

The mean age of the 160 ASPRA participants with data from both the K-PASE and IPAQ short form was 75.1 years $(\mathrm{SD}, 6.3)$ and $62(38.8 \%)$ were men (Table 1). A total of 104 individuals (65.0\%) were currently working and 90 (56.3\%) were engaged in agriculture. Geriatric conditions were prevalent: 62 participants $(38.8 \%)$ with decreased handgrip strength, $60(37.5 \%)$ with multimorbidity, $55(34.4 \%)$ with low cognition, and $54(33.8 \%)$ with sarcopenia according to the AWGS criteria.

\section{Use of the K-PASE vs. IPAQ Short Form for Assessment of Physical Activity Level}

Physical activity level measured using the K-PASE seemed to follow a normal distribution (Fig. 1), with a mean K-PASE total score 149.2 (SD, 76.3). Participants spend their week sitting for 18.2 hours (SD, 10.7), walking for 10.1 hours (SD, 10.1), and doing light sport activity for 2.0 hours (SD, 5.5), moderate sport activity for 0.3 hours (SD, 1.6), strenuous sport activity for 0.7 hours $(S D, 2.8)$, muscle strengthening for 0.1 hour (SD, 0.6), and paid or volunteer work for 18.6 hours (SD, 19.9).

Physical activity level measured using the IPAO short form showed a highly skewed distribution (Fig. 1), with a mean total activity level of 1,524.6 MET-min/wk (SD, 2,089.5). Participants spend their week sitting for 50.2 hours (SD, 24.5), walking for 5.1 hours (SD, 5.2), and doing moderate activity 
for 2.1 hours (SD, 6.3) and vigorous activity for 0 hour (SD, 0.2).

There was a statistically significant, modest correlation between continuous total K-PASE score and continuous MET-min/wk calculated from the IPAQ short form (Spearman correlation coefficient, $0.250, p=0.001$ ). The agreement and correlation between the 2 physical activity instruments were modest with a kappa statistic of $0.286(p<0.001)$. Forty participants $(25 \%)$ were classified as having low activity using the IPAO short form, and $32(20 \%)$ using the K-PASE. Low physical activity level was correlated with most geriatric conditions, regardless of how it was assessed, with some exceptions (Table 2). Depressive mood was statistically significantly correlated with low activity level measured by the K-PASE, but fall history, multimorbidity, and polypharmacy were statistically significantly correlated with low activity level measured using the IPAO short form.

\section{Choice of Physical Activity Questionnaire to Define CHS Frailty Status}

Despite the modest correlation between the K-PASE and IPAO short form, the agreement was higher between the frailty definition based on the K-PASE (CHS-PASE) and the frailty definition based on the IPAQ short form (CHS-IPAQ) (Table 3). Cohen kappa was $0.625(p<0.001)$, which indicates moderate to high agreement ${ }^{25)}$, and Spearman correlation coefficient was $0.564(p<0.001)$. We also developed a logistic model that estimates low physical activity status according to the IPAO short form using the K-PASE items (Supplementary Table 1). When frailty was defined based on the predicted
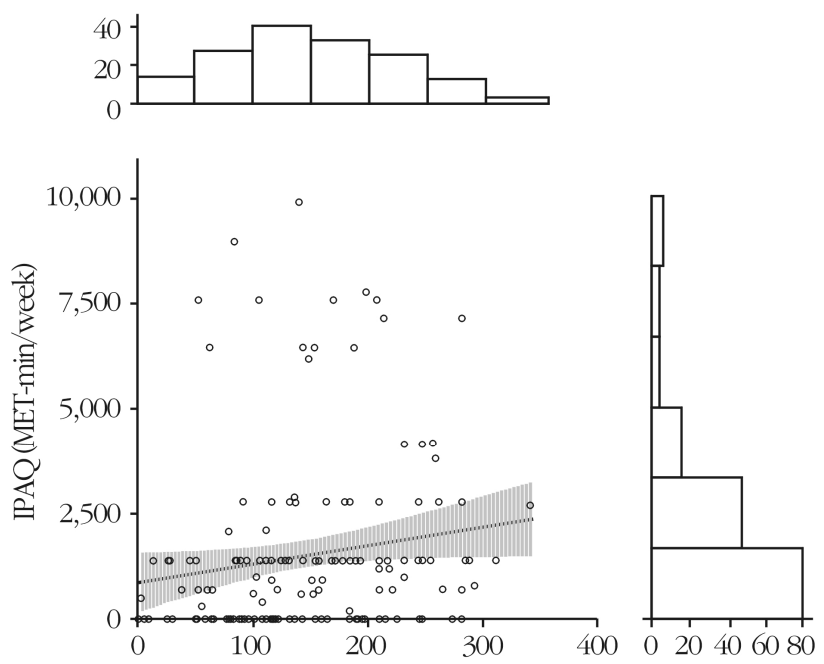

Fig. 1. Comparison of distributions between K-PASE score and energy expenditure calculated from the IPAQ. The line and gray band denote the predicted line by linear regression and standard errors, respectively. K-PASE, Korean Physical Activity Scale of the Elderly; IPAQ, International Physical Activity Questionnaire; MET-min/wk, metabolic equivalent task minutes per week. low activity level from the K-PASE (CHS-IPAQPASE), the agreement and correlation between the frailty definition (CHSIPAQ $\left.{ }_{\text {PASE }}\right)$ and the definition based on the IPAQ short form (CHS-IPAQ) were moderate to high, with a Cohen kappa of 0.711 and Spearman correlation coefficient of $0.598(p<0.001)$. Both the CHS-PASE and CHS-IPAO were positively correlated with most geriatric conditions, except for multimorbidity and polypharmacy (Table 4).

Table 1. Assessment of physical activity by the K-PASE or IPAQ short form in the Aging Study of Pyeongchang Rural Area cohort

\begin{tabular}{|c|c|}
\hline Characteristic & Value \\
\hline Sample size & 160 \\
\hline Age (yr) & $75.1 \pm 6.3$ \\
\hline Men & $62(38.8)$ \\
\hline Educational level (yr) & $4.8 \pm 5.2$ \\
\hline Currently working & $104(65.0)$ \\
\hline Engaged in agriculture & $90(56.3)$ \\
\hline Sarcopenia & $54(33.8)$ \\
\hline Dismobility & $40(25.0)$ \\
\hline Muscle weakness & $62(38.8)$ \\
\hline ADL disability & $23(14.4)$ \\
\hline IADL disability & $43(26.9)$ \\
\hline Cognitive dysfunction & $55(34.4)$ \\
\hline Depression & $26(16.3)$ \\
\hline Fall in the past year & $30(18.8)$ \\
\hline At risk of malnutrition & $88(55.0)$ \\
\hline Multimorbidity & $60(37.5)$ \\
\hline Polypharmacy & $16(10.0)$ \\
\hline Incontinence & $14(8.8)$ \\
\hline \multicolumn{2}{|l|}{ K-PASE } \\
\hline Total physical activity score & $149.2 \pm 76.3$ \\
\hline Sitting, hr/wk (1) & $18.2 \pm 10.7$ \\
\hline Walking, hr/wk (2) & $10.1 \pm 10.1$ \\
\hline Light sport activity, hr/wk (3) & $2.0 \pm 5.5$ \\
\hline Moderate sport activity, hr/wk (4) & $0.3 \pm 1.6$ \\
\hline Strenuous sport activity, hr/wk (5) & $0.7 \pm 2.8$ \\
\hline Muscle strengthening, hr/wk (6) & $0.1 \pm 0.6$ \\
\hline Paid or volunteer work, hr/wk (7-10) & $18.6 \pm 19.9$ \\
\hline \multicolumn{2}{|l|}{ IPAQ } \\
\hline Total physical activity level, MET-min/wk & $1,524.6 \pm 2,089.5$ \\
\hline Sitting, hr/wk & $50.2 \pm 24.5$ \\
\hline Walking, hr/wk & $5.1 \pm 5.2$ \\
\hline Moderate activity, hr/wk & $2.1 \pm 6.3$ \\
\hline Vigorous activity, hr/wk & $0.0 \pm 0.5$ \\
\hline
\end{tabular}

Values are presented as mean \pm standard deviation or number (\%). K-PASE, Korean Physical Activity Scale of the Elderly; IPAQ, International Physical Activity Questionnaire; ADL, activities of daily living; IADL, instrumental activities of daily living; MET-min/ wk, metabolic equivalent task minutes per week. 
Table 2. Correlations between 2 physical activity questionnaires and geriatric conditions

\begin{tabular}{|c|c|c|c|c|}
\hline \multirow{2}{*}{ Geriatric condition } & \multicolumn{2}{|c|}{ K-PASE } & \multicolumn{2}{|c|}{ IPAQ } \\
\hline & Correlation & p-value & Correlation & p-value \\
\hline Sarcopenia & -0.206 & 0.009 & -0.265 & 0.001 \\
\hline Dismobility & -0.394 & $<0.001$ & -0.318 & $<0.001$ \\
\hline Muscle weakness & -0.375 & $<0.001$ & -0.208 & 0.008 \\
\hline ADL disability & -0.364 & $<0.001$ & -0.196 & 0.013 \\
\hline IADL disability & -0.347 & $<0.001$ & -0.251 & 0.001 \\
\hline Cognitive dysfunction & -0.238 & 0.003 & -0.333 & $<0.001$ \\
\hline Depression & -0.232 & 0.003 & -0.119 & 0.137 \\
\hline Fall in the past year & -0.151 & 0.056 & -0.282 & $<0.001$ \\
\hline At risk of malnutrition & -0.209 & 0.008 & -0.195 & 0.014 \\
\hline Multimorbidity & -0.125 & 0.116 & -0.162 & 0.041 \\
\hline Polypharmacy & -0.022 & 0.786 & -0.197 & 0.012 \\
\hline Incontinence & -0.252 & 0.001 & -0.157 & 0.048 \\
\hline
\end{tabular}

K-PASE, Korean Physical Activity Scale of the Elderly; IPAQ, International Physical Activity Questionnaire; ADL, activities of daily living; IADL, instrumental activities of daily living.

Table 3. Comparisons of CHS frailty status calculated using physical activity items from the IPAQ (CHS-IPAQ) and CHS frailty status using the K-PASE (CHS-PASE) or IPAQ estimated from the K-PASE by a regression equation (CHS-IPAQPASE)

\begin{tabular}{lrrcc}
\hline \hline & \multicolumn{4}{c}{ CHS-IPAQ } \\
\cline { 2 - 5 } & Nonfrail & Frail & Cohen kappa & Spearman rho \\
\hline CHS-PASE & & & & 0.626 \\
Nonfrail & 119 & 11 & 0.625 & - \\
Frail & 8 & 22 & - & 0.598 \\
CHS-IPAQPASE & & & & - \\
Nonfrail & 122 & 9 & 0.720 & 0.59 \\
Frail & 5 & 24 & - & - \\
\hline
\end{tabular}

CHS, Cardiovascular Health Study, IPAQ, International Physical Activity Questionnaire; K-PASE, Korean Physical Activity Scale of the Elderly.

\section{DISCUSSION}

In geriatric research and clinical medicine, evaluating frailty status in older people has become indispensable, and measurement of physical activity is needed as a component of the physical frailty phenotype. In this study, we compared physical activity measures using the K-PASE and IPAQ short form and observed a statistically significant, positive correlation between the 2 measures. In addition, we found moderate to high agreement between CHS frailty phenotype definitions based on the K-PASE or IPAQ short form. These results suggest that the IPAO short form might replace the K-PASE as a component of the CHS frailty phenotype in older Koreans.

There are various approaches to measuring physical activity levels, including self-reported questionnaires, self-reported activity diaries, direct observation, accelerometers, and pedometers $^{26)}$. Among these, self-reported questionnaires have been the most used method in large-scale epidemiological research studies and busy outpatient clinics because of its feasibility for administration and despite its possible inaccuracy due to the recall process and inevitable intervening of subjective judgment. The PASE, a proprietary, widely accepted questionnaire initially developed and validated from 23 cities in Massachusetts in the United States ${ }^{16)}$, includes activities such as chopping down trees and carrying wood as household-related activities, which are less conceivable in environments such as Korea or China ${ }^{27,28)}$, and does not assume that older people might spend their energy engaging in agricultural activities that are, however, inevitable in rural area of developing countries. On the other hand, the IPAQshort form, which has been translated into multiple languages, is less vulnerable to cultural and socioeconomic variation owing to its simplicity and objectivity in questionnaire items $^{29)}$.

Although the IPAQ short form consisted of much fewer items than the K-PASE, the results can be converted easily to standardized quantified units (MET-min/wk), which is one of the most advantageous features of the IPAQ short form. With increasing accessibility for objective, wearable instruments such as an accelerometer to be used in communitybased research studies, these data from the IPAQ short form can be easily compared with data obtained using other objective instruments. In addition, given this compatibility, the IPAO short form can be better used to assess the efficacy of exercise-based interventional studies for older people in various settings.

We observed that the physical activity level assessed by using the K-PASE and by using the IPAQ short form had a modest agreement, possibly owing to the differences in the questionnaires. Despite this discrepancy, frailty status 
Il-Young Jang, et al.

Table 4. Correlations between frailty status, defined based on physical activity items assessed using the K-PASE or IPAQ or estimated IPAQ from K-PASE by regression equation (CHS-IPAQPASE)

\begin{tabular}{|c|c|c|c|c|c|c|}
\hline \multirow{2}{*}{ Geriatric condition } & \multicolumn{2}{|c|}{ Frail vs. nonfrail by K-PASE } & \multicolumn{2}{|c|}{ Frail vs. nonfrail by IPAQ } & \multicolumn{2}{|c|}{ Frail vs. nonfrail by IPAQPASE } \\
\hline & Correlation & p-value & Correlation & p-value & Correlation & p-value \\
\hline Sarcopenia & 0.233 & 0.003 & 0.224 & 0.004 & 0.271 & 0.001 \\
\hline Dismobility & 0.610 & $<0.001$ & 0.491 & $<0.001$ & 0.550 & $<0.001$ \\
\hline Muscle weakness & 0.407 & $<0.001$ & 0.229 & 0.004 & 0.372 & $<0.001$ \\
\hline ADL disability & 0.397 & $<0.001$ & 0.275 & $<0.001$ & 0.346 & $<0.001$ \\
\hline IADL disability & 0.431 & $<0.001$ & 0.248 & 0.002 & 0.353 & $<0.001$ \\
\hline Low cognition & 0.309 & $<0.001$ & 0.278 & $<0.001$ & 0.419 & $<0.001$ \\
\hline Depression & 0.319 & $<0.001$ & 0.227 & 0.004 & 0.227 & 0.001 \\
\hline Fall in the past year & 0.138 & 0.081 & 0.151 & 0.057 & 0.278 & $<0.001$ \\
\hline Malnutrition & 0.306 & $<0.001$ & 0.306 & $<0.001$ & 0.304 & $<0.001$ \\
\hline Multimorbidity & 0.058 & 0.467 & 0.116 & 0.145 & 0.014 & 0.856 \\
\hline Polypharmacy & 0.000 & 1.000 & 0.088 & 0.271 & -0.019 & 0.258 \\
\hline Incontinence & 0.248 & 0.002 & 0.225 & 0.004 & 0.255 & 0.001 \\
\hline
\end{tabular}

K-PASE, Korean Physical Activity Scale of the Elderly; IPAQ, International Physical Activity Questionnaire; ADL, activities of daily living; IADL, instrumental activities of daily living.

according to the CHS-PASE and CHS-IPAO agreed highly with each other. Furthermore, frailty status according to both the CHS-PASE and CHS-IPAQ correlated with common geriatric syndromes including functional impairment and fall history. Therefore, instead of the K-PASE, the IPAQ short form might be used as a component of the CHS phenotype for evaluating frailty status in community-dwelling older people.

To our knowledge, this study is the first to directly compare two commonly used physical activity questionnaires to assess CHS frailty phenotype and common geriatric syndromes. In particular, several nationwide epidemiological studies that used the IPAO short form are currently underway in Korea ${ }^{8,14)}$. Thus, establishing the validity of the IPAQ short form to assess frailty and developing a conversion equation from the K-PASE to the IPAO short form are timely to facilitate interpretations of data in comparison with the findings from these studies.

However, there are limitations in the study. Since our study is based on community-dwelling older people in rural agricultural areas, generalizability to urban-dwelling older people may be limited. In this study, we used the ASPRA cohort-specific 20th percentile cutoff point of the K-PASE owing to lack of a nationwide cutoff point for the older Korean population. Although we compared CHS frailty phenotype definitions based on the ASPRA-specific K-PASE cutoff point vs. the nationwide IPAQ cutoff point, it was reassuring to observe moderate to high agreement between the 2 definitions and a similar correlation between each definition and most geriatric conditions. Furthermore, although a large proportion of the study population is engaged in agriculture, neither the K-PASE nor the IPAO short form has specific items to capture their agricultural work as a physical activity. An objective measurement (e.g., accelerometer) will be useful for more accurate assessment of physical activity. An ongoing nationwide aging cohort, the KFACS, that employs the IPAO short form and a wearable device, is expected to provide additional information on validity ${ }^{8}$. Future research should assess the predictive validity of these tools for adverse health events.

In conclusion, our study provides supporting evidence that the IPAQ short form, a simple and free questionnaire, may replace the K-PASE for assessing CHS frailty phenotype in older Koreans. Our results should inform frailty assessment in various research and clinical settings.

Conflicts of Interest Disclosures: Dr. Dae Hyun Kim is a consultant to Alosa Health, a nonprofit educational organization with no relationship to any drug or device manufacturers. The other authors have no potential conflicts of interest to disclose.

\section{Acknowledgements}

The Aging Study of Pyeongchang Rural Area Intervention Study was funded by Pyeongchang County Hospital, Pyeongchang, Korea. Dr. Hee-Won Jung is supported by a Global PhD Fellowship Program through the National Research Foundation of Korea funded by the Ministry of Education (NRF2015H1A2A1030117). Dr. Chang Ki Lee is supported by a scholarship from the Korean Geriatrics Society in 2015. Dr. Dae Hyun Kim is supported by the Paul B. Beeson Clinical Scientist Development Award in Aging (K08AG051187) from the National Institute on Aging, American Federation for Aging Research, The John A. Hartford Foundation, and The Atlantic Philanthropies. 


\section{Supplementary Material}

Supplementary Table 1 can be found via http://www.e-agmr. org/src/sm/agmr-21-101-s001.pdf.

\section{REFERENCES}

1. Nikolich-Žugich J, Goldman DP, Cohen PR, Cortese D, Fontana L, Kennedy BK, et al. Preparing for an Aging World: Engaging Biogerontologists, Geriatricians, and the Society. J Gerontol A Biol Sci Med Sci 2016;71:435-44.

2. Clegg A, Young J, Iliffe S, Rikkert MO, Rockwood K. Frailty in elderly people. Lancet 2013;381:752-62.

3. Rockwood K, Song X, MacKnight C, Bergman H, Hogan DB, McDowell I, et al. A global clinical measure of fitness and frailty in elderly people. CMAJ 2005;173:489-95.

4. Kim SW, Han HS, Jung HW, Kim KI, Hwang DW, Kang SB, et al. Multidimensional frailty score for the prediction of postoperative mortality risk. JAMA Surg 2014;149:633-40.

5. Jung HW, Kim SW, Ahn S, Lim JY, Han JW, Kim TH, et al. Prevalence and outcomes of frailty in Korean elderly population: comparisons of a multidimensional frailty index with two phenotype models. PLoS One 2014;9:e87958.

6. Fried LP, Tangen CM, Walston J, Newman AB, Hirsch C, Gottdiener J, et al. Frailty in older adults: evidence for a phenotype. J Gerontol A Biol Sci Med Sci 2001;56:M146-56.

7. Jung HW, Jang IY, Lee YS, Lee CK, Cho EI, Kang WY, et al. Prevalence of frailty and aging-related health conditions in older koreans in rural communities: a cross-sectional analysis of the Aging Study of Pyeongchang Rural Area. J Korean Med Sci 2016;31:345-52.

8. Won CW, Lee Y, Choi J, Kim KW, Park Y, Park H, et al. Starting construction of frailty cohort for elderly and intervention study. Ann Geriatr Med Res 2016;20:114-7.

9. Liu H, Graber TG, Ferguson-Stegall L, Thompson LV. Clinically relevant frailty index for mice. J Gerontol A Biol Sci Med Sci 2014;69:1485-91.

10. Cameron ID, Fairhall N, Langron C, Lockwood K, Monaghan $\mathrm{N}$, Aggar C, et al. A multifactorial interdisciplinary intervention reduces frailty in older people: randomized trial. BMC Med 2013;11:65.

11. Jung HW, Kim SW, Lim JY, Kim KW, Jang HC, Kim CH, et al. Frailty status can predict further lean body mass decline in older adults. J Am Geriatr Soc 2014;62:2110-7.

12. Choe MA, Kim J, Jeon MY, Chae YR. Evaluation of the Korean Version of Physical Activity Scale for the Elderly (K-PASE). Korean J Women Health Nurs 2010;16:47-59.

13. Chun MY. Validity and Reliability of Korean Version of International Physical Activity Questionnaire Short Form in the Elderly. Korean J Fam Med 2012;33:144-51.

14. Kim GS, Im E, Rhee JH. Association of physical activity on body composition, cardiometabolic risk factors, and prevalence of cardiovascular disease in the Korean population (from the fifth Korea national health and nutrition examination survey, 2008-2011). BMC Public Health 2017;17:275.

15. Jang IY, Jung HW, Lee CK, Lee YS, Kim KI, Kim KW, et al. Rural and urban disparities in frailty and aging-related health conditions in Korea. J Am Geriatr Soc 2016;64:908-11.

16. Washburn RA, Smith KW, Jette AM, Janney CA. The Physical Activity Scale for the Elderly (PASE): development and evaluation. J Clin Epidemiol 1993;46:153-62.

17. Washburn RA, McAuley E, Katula J, Mihalko SL, Boileau RA. The physical activity scale for the elderly (PASE): evidence for validity. J Clin Epidemiol 1999;52:643-51.

18. Avila-Funes JA, Amieva H, Barberger-Gateau P, Le Goff M, Raoux N, Ritchie K, et al. Cognitive impairment improves the predictive validity of the phenotype of frailty for adverse health outcomes: the three-city study. J Am Geriatr Soc 2009;57:45361.

19. Fukutomi E, Okumiya K, Wada T, Sakamoto R, Ishimoto Y, Kimura $\mathrm{Y}$, et al. Importance of cognitive assessment as part of the "Kihon Checklist" developed by the Japanese Ministry of Health, Labor and Welfare for prediction of frailty at a 2-year follow up. Geriatr Gerontol Int 2013;13:654-62.

20. Chen LK, Liu LK, Woo J, Assantachai P, Auyeung TW, Bahyah KS, et al. Sarcopenia in Asia: consensus report of the Asian Working Group for Sarcopenia. J Am Med Dir Assoc 2014;15: 95-101.

21. Kang Y, Na DL, Hahn S. A validity study on the Korean MiniMental State Examination (K-MMSE) in dementia patients. J Korean Neurol Assoc 1997;15:300-8.

22. Park JH, Kim KW. A review of the epidemiology of depression in Korea. J Korean Med Assoc 2011;54:362-9.

23. Rubenstein LZ, Harker JO, Salvà A, Guigoz Y, Vellas B. Screening for undernutrition in geriatric practice: developing the short-form mini-nutritional assessment (MNA-SF). J Gerontol A Biol Sci Med Sci 2001;56:M366-72.

24. Youden WJ. Index for rating diagnostic tests. Cancer 1950;3: 32-5.

25. McHugh ML. Interrater reliability: the kappa statistic. Biochem Med (Zagreb) 2012;22:276-82.

26. Sylvia LG, Bernstein EE, Hubbard JL, Keating L, Anderson EJ. Practical guide to measuring physical activity. J Acad Nutr Diet 2014;114:199-208.

27. Vaughan K, Miller WC. Validity and reliability of the Chinese translation of the Physical Activity Scale for the Elderly (PASE). Disabil Rehabil 2013;35:191-7.

28. Son KY, Cho B, Kwon IS, Park BJ, Sun Woo D, Yoon JL, et al. The Korean Physical Activity Questionnaire for Elderly (PAQE): Development and Evaluation of Validity and Reliability. J Korean Geriatr Soc 2012;16:55-65.

29. Craig CL, Marshall AL, Sjöström M, Bauman AE, Booth ML, Ainsworth BE, et al. International physical activity questionnaire: 12 -country reliability and validity. Med Sci Sports Exerc 2003;35:1381-95. 